\title{
An Approach to Solve Multi Attribute Decision-making Problem Based on the New Possibility Measure of Picture Fuzzy Numbers
}

\author{
K. Deva, S. Mohanaselvi* \\ Department of Mathematics, College of Engineering and technology, SRM Institute of Science and Technology, India
}

\begin{abstract}
Received September 8, 2021; Revised November 25, 2021; Accepted December 13, 2021
Cite This Paper in the following Citation Styles

(a): [1] K. Deva, S. Mohanaselv, ”An Approach to Solve Multi Attribute Decision-making Problem Based on the New Possibility Measure of Picture Fuzzy Numbers," Mathematics and Statistics, Vol.10, No.1, pp. 153-159, 2022. DOI: 10.13189/ms.2022.100113

(b): K. Deva, S. Mohanaselv, (2022). An Approach to Solve Multi Attribute Decision-making Problem Based on the New Possibility Measure of Picture Fuzzy Numbers. Mathematics and Statistics, 10(1), 153-159. DOI: 10.13189/ms.2022.100113
\end{abstract}

Copyright $@ 2022$ by authors, all rights reserved. Authors agree that this article remains permanently open access under the terms of the Creative Commons Attribution License 4.0 International License

\begin{abstract}
A picture fuzzy set is a more powerful tool to deal with uncertainties in the given informa- tion as compared to fuzzy set and intuitionistic fuzzy set and has energetic applications in decision-making. The aim of this study is to develop a new possibility measure for ranking picture fuzzy numbers and then some of its basic properties are proved. The proposed method provides the same ranking order as the score function in the literature. Moreover, the new possibility measure can provide additional information for the relative comparison of the picture fuzzy numbers. A picture fuzzy multi attribute decision-making problem is solved based on the possibility matrix generated by the proposed method after being aggregated using picture fuzzy Einstein weighted averaging aggregation operator. To verify the importance of the proposed method, an picture fuzzy multi attribute decisionmaking strategy is presented along with an application for selecting suitable alternative. The superiority of the proposed method and limitations of the existing methods are discussed with the help of a comparative study. Finally, a numerical example and comparative analysis are provided to illustrate the practicality and feasibility of the proposed method.
\end{abstract}

Keywords Picture Fuzzy Number, Aggregation Operator, New Possibility Measure, Multi Attribute Decision-making

\section{Introduction}

Multi attribute decision-making (MADM) problems are an important part of decision theory in which the optimal alternative is chosen from the different alternatives. The fundamental concept of fuzzy set (FS) theory was initially developed by Zadeh [1] in 1965. In 1986, Atanassov [2] implemented the intuitionistic fuzzy set (IFS) as an extension of the FS. Picture fuzzy set (PFS) is a direct extension of IFS, as proposed by Cuong $[3,4]$ who also described some operations on PFS. Phong [5] investigated some properties of compositions of picture fuzzy (PF) relations. Singh [6] proposed a correlation coefficient for PFSs and used it for clustering analysis in the $\mathrm{PF}$ environment. Two correlation coefficients of PFSs, as well as some of their properties were suggested by Ganie [7]. Wei [8] discussed a MADM problem based on the PF weighted cross-entropy as an extension of the cross-entropy of PFSs, which is used to rank the different alternatives. Wang [9] developed some geometric aggregation operators under the PFSs. Dutta [10] introduced the equivalence of PF relation based on $(\alpha, \beta, \gamma)-$ cuts. Shovan Dogra [11] The notions of $\mathrm{PF}$ matrix, restricted PF matrix and special restricted PF matrix are established. Amalendu Si [12] proposed a decision-making approach for preferable medicine selection using PFS, Dempster-Shafer theory of evidence and grey relational analysis. Wei [13] introduced arithmetic and geometric operations to develop some PF aggregation operators and also studied their application in MADM problem. Garg [14] proposed some aggregation operators under PF environment, their desirable properties and their application in MADM problem. Wei [15] 
developed some picture fuzzy aggregation operators on the basic of the traditional arithmetic, geometric operations and Hamacher operations and utilized these operators to develop some approaches to solve the picture fuzzy MADM. Khan [16] examined the MADM problem based on the arithmetic aggregation operators under PF environment with Einstein t-norms and t-conorms. Qiyas [17] generated the Concept of Yager operators with the picture fuzzy set environment and its application to emergency program selection. Cuong [18,19] investigated the classification of representable picture t-norms, picture t-conorms operators and PF logic operators.

It is very important for PF decision-making to rate PFS. Thus, a comprehensive literature review survey of many research studies on the PF environment. The present research study aims to improve the New possibility measure for picture fuzzy numbers (PFNs). The possibility theory is a mathematical concept of dealing with several kinds of ambiguities and is a superior alternative to the probability theory that uses the possibility measure between 0 and 1. Zadeh [20] discussed the theory of possibility with the concept of possibility and probability in order to understand the role of human ability to interpret possibilities to imprecisely defined problems in a fuzzy environment. Wenxiu [21] proposed the extension principle through the extended fuzzy operators with t-norms and tconorms for a new ordering and also introduced sigma-algebra to define fuzzy truth-valued possibility. Wang [22] developed the estimation of utility intervals with aggregation method associated with linear programming and simple additive weighting method to generate an aggregation ranking, which demonstrated the validity and potential applications of the proposed method. Van Dinh et al. [23] presented his ideas on difference and dissimilarity between two PFSs; the authors also studied, the application of dissimilarity measure in MADM. Garg [24] developed a possibility degree measure for ranking IFNs and also presented, some shortcomings of the existing possibility degree method and score function of intuitionistic fuzzy numbers.

In this study, a new possibility measure is proposed for ranking PFNs by extending the possibility measure of intuitionistic fuzzy (IF) environment [24-27]. The present study demonstrates that the same ranking can be obtained by both the new possibility measure and current approaches. Meanwhile, ranking result based on the new possibility measure will indicate additional information for the relative comparison of the PFNs.

The motivations of this article are outlined as follows:

(i) A new possibility measure for ranking PFNs is proposed by extending the possibility measure of the IF environment [24-27].

(ii) The proposed new possibility measure deal with the relative comparison of the PFNs.

(iii) The proposed method give more accurate and exact choice values in decision results when applied to MADM problem (iv) The relative comparisons are more realistic in terms of knowing how good or bad an alternative is when compared to the rest of the alternatives on the multi attribute evaluation.

The contributions of this article are outlined as follows:

(i) The new possibility measure is proposed.

(ii) An algorithm for MADM problem using the proposed method is described and an application is presented to show the method's applicability in the real world.

(iii) The proposed method is demonstrated by a comparative study with other existing approaches.

The present research article is structured into seven comprehensive sections. Some of the basic definitions of PFSs and picture fuzzy Einstein weighted averaging (PFEWA) operator are outline in Section 2. The newly proposed possibility measure is covered under Section 3. Section 4 discusses a method of decision-making based on the proposed new possibility measure to solve the problem of MADM in the PF environment. A numerical example is given in Section 5 to establish the validity of this study. In Section 6, a comparative analysis between the existing methods and the proposed method is reviewed. In the final Section, the conclusions drawn from the present study are detailed.

\section{Preliminaries}

In this section, the basic concept of picture fuzzy set has been reviewed.

Definition 2.1: [3] A picture fuzzy set $A$ on universal set $X$ is defined by

$$
\mathrm{A}=\left\{\left(x, \mu_{A}(x), \eta_{A}(x), \nu_{A}(x)\right) \mid x \in X\right\}
$$

where $\mu_{A}(x) \in[0,1], \eta_{A}(x) \in[0,1]$ and $\nu_{A}(x) \in[0,1]$ are the degree of membership, the degree of neutral membership and the degree of non-membership of $x \in A$ such that $0 \leq \mu_{A}(x)+\eta_{A}(x)+\nu_{A}(x) \leq 1 \forall x \in X$.

The degree of refusal membership is given by for $x \in X, \pi_{A}(x)=1-\mu_{A}(x)-\eta_{A}(x)-\nu_{A}(x)$.

Definition 2.2: [14] For convenience, let us denote $\alpha=$ $\left(\mu_{\alpha}, \eta_{\alpha}, \nu_{\alpha}\right)$ as a picture fuzzy number (PFN), and its score function $S(\alpha)$ is defined by

$$
S(\alpha)=\mu_{\alpha}-\eta_{\alpha}-\nu_{\alpha} ; S(\alpha) \in[-1,1] .
$$

Definition 2.3: [14] Let $\alpha=\left(\mu_{\alpha}, \eta_{\alpha}, \nu_{\alpha}\right)$ be a PFN and its accuracy function $H(\alpha)$ is defined by

$$
H(\alpha)=\mu_{\alpha}+\eta_{\alpha}+\nu_{\alpha} ; H(\alpha) \in[0,1] .
$$

Definition 2.4: [14] Let $\alpha_{1}=\left(\mu_{\alpha_{1}}, \eta_{\alpha_{1}}, \nu_{\alpha_{1}}\right)$ and $\alpha_{2}=$ $\left(\mu_{\alpha_{2}}, \eta_{\alpha_{2}}, \nu_{\alpha_{2}}\right)$ be two PFNs. then the following comparison rules can be used. 
Table 1. Comparative analysis between some existing methods and the proposed approach

\begin{tabular}{lllll}
\hline Reference Article & Weight information & Aggregation operators & Ranking method & Relative comparison \\
\hline Wang (2017) & Known & Algebraic & Score function & $\times$ \\
Garg (2017) & Known & Archimedean & Score function & $\times$ \\
Wei (2017) & Known & Algebraic & Score function & $\times$ \\
Wei (2018) & Known & Hamacher & Score function & $\times$ \\
Khan (2019) & Known & Einstein Averaging & Score function & $\times$ \\
Qiyas (2020) & Known & Yager operators & Score function & $\times$ \\
Proposed Method & Known & Einstein Averaging & New possibility measure & $\checkmark$ \\
\hline
\end{tabular}

(i) If $S\left(\alpha_{1}\right)>S\left(\alpha_{2}\right)$, then $\alpha_{1}>\alpha_{2}$;

(ii) If $S\left(\alpha_{1}\right)=S\left(\alpha_{2}\right)$, then
(a) If $H\left(\alpha_{1}\right)>H\left(\alpha_{2}\right)$, then $\alpha_{1}>\alpha_{2}$;
(b) If $H\left(\alpha_{1}\right)=H\left(\alpha_{2}\right)$, then $\alpha_{1}=\alpha_{2}$.

Definition 2.5: [14] Let $\alpha_{i}=\left\langle\mu_{\alpha_{i}}, \eta_{\alpha_{i}}, \nu_{\alpha_{i}}\right\rangle,(i=$ $1,2, \ldots, n)$ be family of PFNs an picture fuzzy Einstein weighted arithmetic aggregation operator is defined as,

$$
\begin{aligned}
& P F E W A\left(\alpha_{1}, \alpha_{2}, \ldots, \alpha_{n}\right)=w_{1} \alpha_{1} \oplus w_{2} \alpha_{2} \oplus \ldots \oplus w_{n} \alpha_{n} \\
& =\left\langle\begin{array}{l}
\frac{\prod_{i=1}^{n}\left(1+\mu_{\alpha_{i}}\right)^{w_{i}}-\prod_{i=1}^{n}\left(1-\mu_{\alpha_{i}}\right)^{w_{i}}}{\prod_{i=1}^{n}\left(1+\mu_{\alpha_{i}}\right)^{w_{i}}+\prod_{i=1}^{n}\left(1-\mu_{\alpha_{i}}\right)^{w_{i}}}, \\
\frac{2 \prod_{i=1}^{n}\left(\eta_{\alpha_{i}}\right)^{w_{i}}}{\prod_{i=1}^{n}\left(2-\eta_{\alpha_{i}}\right)^{w_{i}}+\prod_{i=1}^{n}\left(\eta_{\alpha_{i}}\right)^{w_{i}}},
\end{array}\right. \\
& \frac{2 \prod_{i=1}^{n}\left(\nu_{\alpha_{i}}\right)^{w_{i}}}{\prod_{i=1}^{n}\left(2-\nu_{\alpha_{i}}\right)^{w_{i}}+\prod_{i=1}^{n}\left(\nu_{\alpha_{i}}\right)^{w_{i}}}
\end{aligned}
$$

where $w=\left(w_{1}, w_{2}, \ldots, w_{n}\right)^{T}$ be the weighing vector" of $\alpha_{i}(i=1,2,3, \ldots, n)$ with $w_{i} \in[0,1]$ and $\sum_{i=1}^{n} w_{i}=1$.

\section{New Possibility measure for Picture fuzzy numbers}

A new possibility measure is proposed in this section to the rank of PFNs $\alpha_{i}=\left\langle\mu_{\alpha_{i}}, \eta_{\alpha_{i}}, \nu_{\alpha_{i}}\right\rangle,(i=1,2, \ldots, n)$.

Definition 3.1: Let $\alpha_{1}=\left\langle\mu_{\alpha_{1}}, \eta_{\alpha_{1}}, \nu_{\alpha_{1}}\right\rangle$ and $\alpha_{2}=\left\langle\mu_{\alpha_{2}}, \eta_{\alpha_{2}}, \nu_{\alpha_{2}}\right\rangle$ be two PFNs, then the new possibility measure $p\left(\alpha_{1} \succeq \alpha_{2}\right)$ for $\alpha_{1} \succeq \alpha_{2}$ is proposed as,

$$
\begin{aligned}
& p\left(\alpha_{1} \succeq \alpha_{2}\right) \\
& =\min \left(\max \left(\frac{2\left(\left(\mu_{\alpha_{1}}+\pi_{\alpha_{1}}\right)-\mu_{\alpha_{2}}\right)+\pi_{\alpha_{2}}}{3\left(\pi_{\alpha_{1}}+\pi_{\alpha_{2}}\right)}, 0\right), 1\right)
\end{aligned}
$$

Where, either $\pi_{\alpha_{1}} \neq 0$ or $\pi_{\alpha_{2}} \neq 0$. On the other hand, if $\pi_{\alpha_{1}}=\pi_{\alpha_{2}}=0$, then

$$
p\left(\alpha_{1} \succeq \alpha_{2}\right)= \begin{cases}1 ; & \mu_{\alpha_{1}}>\mu_{\alpha_{2}} \\ 0 ; & \mu_{\alpha_{1}}<\mu_{\alpha_{2}} \\ 0.5 ; & \mu_{\alpha_{1}}=\mu_{\alpha_{2}}\end{cases}
$$

Theorem 3.1: Let $\alpha_{1}$ and $\alpha_{2}$ be two PFNs, then

(i) $0 \leq p\left(\alpha_{1} \succeq \alpha_{2}\right) \leq 1$;

(ii) $p\left(\alpha_{1} \succeq \alpha_{2}\right)=0.5$ if $\alpha_{1}=\alpha_{2}$;

(iii) $p\left(\alpha_{1} \succeq \alpha_{2}\right)+p\left(\alpha_{2} \succeq \alpha_{1}\right)=1$

Proof:

(i) $p\left(\alpha_{1} \succeq \alpha_{2}\right) \leq 0$ is evidently, so we need to prove only $p\left(\alpha_{1} \succeq \alpha_{2}\right) \leq 1$. for it, we take

$a=\frac{2\left(\left(\mu_{\alpha_{1}}+\pi_{\alpha_{1}}\right)-\mu_{\alpha_{2}}\right)+\pi_{\alpha_{2}}}{3\left(\pi_{\alpha_{1}}+\pi_{\alpha_{2}}\right)}$

Then, the following cases are discussed:

(a) If $a \geq 1$,then

$$
\begin{gathered}
p\left(\alpha_{1} \succeq \alpha_{2}\right)=\min (\max (a, 0), 1) \\
=1 \\
\text { (b) If } 0<a<1 \text {, then } \\
p\left(\alpha_{1} \succeq \alpha_{2}\right)=\min (\max (a, 0), 1) \\
=a
\end{gathered}
$$

(c) If $a \leq 0$,then

$$
\begin{aligned}
p\left(\alpha_{1} \succeq \alpha_{2}\right) & =\min (\max (a, 0), 1) \\
& =0
\end{aligned}
$$

Hence, in all cases,

we have $0 \leq p\left(\alpha_{1} \succeq \alpha_{2}\right) \leq 1$.

(ii) Let $\alpha_{1}=\left\langle\mu_{\alpha_{1}}, \eta_{\alpha_{1}}, \nu_{\alpha_{1}}\right\rangle$ and $\alpha_{2}=\left\langle\mu_{\alpha_{2}}, \eta_{\alpha_{2}}, \nu_{\alpha_{2}}\right\rangle$ be two PFNs. If $\alpha_{1}=\alpha_{2}$, which implies that $\mu_{\alpha_{1}}=\mu_{\alpha_{2}}, \eta_{\alpha_{1}}=\eta_{\alpha_{2}}$ and $\nu_{\alpha_{1}}=\nu_{\alpha_{2}}$. then Equation (2) become 


$$
\begin{aligned}
& p\left(\alpha_{1} \succeq \alpha_{2}\right) \\
& =\min \left(\max \left(\frac{2\left(\left(\mu_{\alpha_{1}}+\pi_{\alpha_{1}}\right)-\mu_{\alpha_{2}}\right)+\pi_{\alpha_{2}}}{3\left(\pi_{\alpha_{1}}+\pi_{\alpha_{2}}\right)}, 0\right), 1\right) \\
& =\min \left(\max \left(\frac{2\left(\left(\mu_{\alpha_{1}}+\pi_{\alpha_{1}}\right)-\mu_{\alpha_{1}}\right)+\pi_{\alpha_{1}}}{3\left(\pi_{\alpha_{1}}+\pi_{\alpha_{1}}\right)}, 0\right), 1\right) \\
& =\min \left(\max \left(\frac{3 \pi_{\alpha_{1}}}{6 \pi_{\alpha_{1}}}, 0\right), 1\right) \\
& =\min (\max (0.5,0), 1) \\
& =0.5
\end{aligned}
$$

(iii) For two PFNs $\alpha_{1}=\left\langle\mu_{\alpha_{1}}, \eta_{\alpha_{1}}, \nu_{\alpha_{1}}\right\rangle$ and $\alpha_{2}=\left\langle\mu_{\alpha_{2}}, \eta_{\alpha_{2}}, \nu_{\alpha_{2}}\right\rangle$ we take

$$
\begin{aligned}
& a=\frac{2\left(\left(\mu_{\alpha_{1}}+\pi_{\alpha_{1}}\right)-\mu_{\alpha_{2}}\right)+\pi_{\alpha_{2}}}{3\left(\pi_{\alpha_{1}}+\pi_{\alpha_{2}}\right)} \\
& b=\frac{2\left(\left(\mu_{\alpha_{2}}+\pi_{\alpha_{2}}\right)-\mu_{\alpha_{1}}\right)+\pi_{\alpha_{1}}}{3\left(\pi_{\alpha_{2}}+\pi_{\alpha_{1}}\right)}
\end{aligned}
$$

Such that

$$
\begin{aligned}
& a+b \\
& =\frac{2\left(\left(\mu_{\alpha_{1}}+\pi_{\alpha_{1}}\right)-\mu_{\alpha_{2}}\right)+\pi_{\alpha_{2}}+2\left(\left(\mu_{\alpha_{2}}+\pi_{\alpha_{2}}\right)-\mu_{\alpha_{1}}\right)+\pi_{\alpha_{1}}}{3\left(\pi_{\alpha_{2}}+\pi_{\alpha_{1}}\right)} \\
& =\frac{2 \mu_{\alpha_{1}}+2 \pi_{\alpha_{1}}-2 \mu_{\alpha_{2}}+\pi_{\alpha_{2}}+2 \mu_{\alpha_{2}}+2 \pi_{\alpha_{2}}-2 \mu_{\alpha_{1}}+\pi_{\alpha_{1}}}{3\left(\pi_{\alpha_{2}}+\pi_{\alpha_{1}}\right)} \\
& =\frac{3\left(\pi_{\alpha_{2}}+\pi_{\alpha_{1}}\right)}{3\left(\pi_{\alpha_{2}}+\pi_{\alpha_{1}}\right)} \\
& =1 .
\end{aligned}
$$

Then, for the following cases:

(a) If $a \leq 0$ and $b \leq 0$ then

$p\left(\alpha_{1} \succeq \alpha_{2}\right)+p\left(\alpha_{2} \succeq \alpha_{1}\right)$

$=\min (\max (a, 0), 1)+\min (\max (b, 0), 1)$

$=0+1$

$=1$.

(b) If $0<a, b<1$ then

$p\left(\alpha_{1} \succeq \alpha_{2}\right)+p\left(\alpha_{2} \succeq \alpha_{1}\right)$

$=\min (\max (a, 0), 1)+\min (\max (b, 0), 1)$

$=a+b$

$=1$.

(c) If $a \geq 1$ and then

$p\left(\alpha_{1} \succeq \alpha_{2}\right)+p\left(\alpha_{2} \succeq \alpha_{1}\right)$

$=\min (\max (a, 0), 1)+\min (\max (b, 0), 1)$

$=1+0$

$=1$.

We have from all the cases $p\left(\alpha_{1} \succeq \alpha_{2}\right)+p\left(\alpha_{2} \succeq \alpha_{1}\right)=1$.
Example 3.1: Let $\alpha_{1}=\langle 0.2,0.5,0.1\rangle$ and $\alpha_{2}=$ $\langle 0.3,0.1,0.3\rangle$ be two PFNs then

$$
\begin{aligned}
p\left(\alpha_{1} \succeq \alpha_{2}\right) & =\min \left(\max \left(\frac{2((0.2+0.2)-0.3)+0.3}{3(0.2+0.3)}, 0\right), 1\right) \\
& =\min (\max (0.333,0), 1) \\
& =0.333 .
\end{aligned}
$$

In addition, $p=\left(p_{k j}\right)_{n \times n}$ is the possibility matrix, where $p_{k j}=p\left(\alpha_{k} \succeq \alpha_{j}\right) ; k, j \in(1,2, \ldots, n)$. Thus,

$$
P=\left(\begin{array}{cccc}
p_{11} & p_{12} & \cdots & p_{1 n} \\
p_{21} & p_{22} & \cdots & p_{2 n} \\
\vdots & \vdots & \ddots & \vdots \\
p_{n 1} & p_{n 2} & \cdots & p_{n n}
\end{array}\right)
$$

Determine the alternatives ranking order, according to the decreasing order of $\alpha_{k} ; k \in(1,2, \ldots, n)$ [27] defined as

$$
r_{k}=\frac{1}{n(n-1)}\left(\sum_{j=1}^{n} p_{k j}+\frac{n}{2}-1\right) .
$$

Then, the values of $\alpha_{k}$ are in degreasing order with the ranking order of all $\alpha_{k} ; k \in(1,2, \ldots, n)$ alternatives.

Example 3.2: Consider $\alpha_{1}=\langle 0.5,0.1,0.3\rangle$ and $\alpha_{2}=\langle 0.4,0.3,0.2\rangle$ which are two PFNs that are equal in score value. The new possibility measure is applied to this numbers,

$$
\begin{aligned}
& \qquad \begin{aligned}
p\left(\alpha_{1} \succeq \alpha_{2}\right) & =\min \left(\max \left(\frac{2((0.5+0.1)-0.4)+0.1}{3(0.1+0.1)}, 0\right), 1\right) \\
& =\min (\max (0.833,0), 1) \\
& =0.833 .
\end{aligned} \\
& \text { And } p\left(\alpha_{2} \succeq \alpha_{1}\right)=0.167
\end{aligned}
$$

Therefore, using Equation (5), the possibility matrix is formulated as,

$$
P=\left(\begin{array}{ll}
0.500 & 0.833 \\
0.167 & 0.500
\end{array}\right)
$$

The ranking value for the PFNs $\alpha_{k}(k=1,2)$ is thus determined using Equation (7), Here, $r_{1}=0.665$ and $r_{2}=0.335$. since $r_{1}>r_{2}$, thus we get $\alpha_{1} \stackrel{0.833}{\succ} \alpha_{2}$.

\section{Application of new possibility mea- sure to the multi-attribute decision making}

In this section, we present a MADM problem based on new possibility measure under PF environment. The decision-making problem consists of ' $m$ ' various alternatives $S_{1}, S_{2}, \ldots, S_{m}$ which are evaluated by a decision maker under the set of ' $n$ ' various attributes $T_{1}, T_{2}, \ldots, T_{n}$ which 


$$
\begin{aligned}
& S_{1}=\langle 0.413,0.218,0.227\rangle, \\
& S_{2}=\langle 0.600,0.200,0.142\rangle, \\
& S_{3}=\langle 0.430,0.220,0.218\rangle, \\
& S_{4}=\langle 0.254,0.215,0.338\rangle .
\end{aligned}
$$

Step 3: The possibility matrix obtained by pairwise comparison of these PFNs using Equation (10), is given by

$$
P=\left(\begin{array}{cccc}
0.50 & 0.00 & 0.46 & 0.79 \\
1.00 & 0.50 & 1.00 & 1.00 \\
0.54 & 0.00 & 0.50 & 0.83 \\
0.21 & 0.00 & 0.17 & 0.50
\end{array}\right)
$$

Step 4: $S_{k}(k=1,2,3,4)$ is computed by Equation $(12)$ and is found that $r_{1}=0.229, r_{2}=0.375, r_{3}=0.239$ and $r_{4}=0.156$. Consequently, the ranking of the alternatives is as follows, $S_{2} \stackrel{1.00}{\succ} S_{3} \stackrel{0.54}{\succ} S_{1} \stackrel{0.79}{\succ} S_{4}$.

\section{Comparative analysis}

This section consists of the comparative analysis of several existing methods with proposed method are shown in the Table 4.

Table 4. Comparison with existing approaches

\begin{tabular}{ll}
\hline Existing operators & Ranking \\
\hline PFWG [13] & $S_{2} \succ S_{3} \succ S_{1} \succ S_{4}$ \\
PFOWG [13] & $S_{2} \succ S_{3} \succ S_{1} \succ S_{4}$ \\
PFHA [13] & $S_{2} \succ S_{3} \succ S_{1} \succ S_{4}$ \\
PFHG [13] & $S_{2} \succ S_{3} \succ S_{1} \succ S_{4}$ \\
PFWA [14] & $S_{2} \succ S_{3} \succ S_{1} \succ S_{4}$ \\
PFOWA [14] & $S_{2} \succ S_{3} \succ S_{1} \succ S_{4}$ \\
PFHA [14] & $S_{2} \succ S_{1} \succ S_{3} \succ S_{4}$ \\
PFHWA [15] & $S_{2} \succ S_{1} \succ S_{3} \succ S_{4}$ \\
PFHWG [15] & $S_{2} \succ S_{1} \succ S_{3} \succ S_{4}$ \\
PFEWA [16] & $S_{2} \succ S_{3} \succ S_{1} \succ S_{4}$ \\
PFYWAA [17] & $S_{2} \succ S_{3} \succ S_{1} \succ S_{4}$ \\
PFYWGA [17] & $S_{2} \succ S_{3} \succ S_{1} \succ S_{4}$ \\
\hline Proposed Method & $S_{2} 1.00 S_{3}{ }^{0.54} S_{1}{ }^{0.79} S_{4}$ \\
\hline
\end{tabular}

According to Table 4, the rank is same by using our new possibility measure and the existing approaches. While the proposed method provides additional information for the relative comparison to the decision makers, where $S_{2}$ alternative is more superior to $S_{3}$ than $S_{1}$ superior to $S_{4}$.

\section{Conclusions}

In this research study, a new possibility measure for ranking PFNs is proposed. This method brings the same ranking order of PFNs that is derived by the score function in the literature. Furthermore, the proposed new possibility measure can provide relative comparison of the PFNs. The proposed new possibility measure generates a possibility matrix, which is used to build a basic method for solving picture fuzzy multi attribute decision-making problems. After aggregating a decision matrix with the picture fuzzy Einstein weighted averaging aggregation operator, the proposed method is applied to it. An illustrative example is used to demonstrate the developed approach, and the result is compared with some of the current ranking approaches in the PF environment. In future work, we shall continue working in the extension and application of the possibility measure to the other domain [28-30].

\section{Acknowledgements}

We are very grateful to experts for their appropriate and constructive suggestions to improve this template.

\section{REFERENCES}

[1] L. Zadeh, "Fuzzy Sets," Inf. Control, vol. 8, pp. 338-353, 1965, DOI: 10.1016/S0019-9958(65)90241-X.

[2] Atanassov, K.T, “Intuitionistic fuzzy sets," Fuzzy Sets Syst, Vol. 20, pp. 87-96, 1986.

[3] Cuong, Bui Cong, and Vladik Kreinovich, "Picture Fuzzy Setsa new concept for computational intelligence problems," In 2013 third world congress on information and communication technologies (WICT 2013), pp. 1-6. IEEE, 2013.

[4] Cuong, Bui Cong, and V. Kreinovich, "Picture fuzzy sets." Journal of Computer Science and Cybernetics, vol. 30, no. 4, pp. 409-420, 2014. DOI: 10.15625/1813-9663/30/4/5032.

[5] Phong, Pham Hong, Dinh Trong Hieu, R. T. Ngan, and Pham Thi Them, "Some compositions of picture fuzzy relations," In Proceedings of the 7th national conference on fundamental and applied information technology research (FAIR'7), Thai Nguyen, pp. 19-20, 2014.

[6] Singh, Pushpinder, "Correlation coefficients for picture fuzzy sets," Journal of Intelligent \& Fuzzy Systems, vol. 28, no. 2, pp. 591-604, 2015. DOI: 10.3233/IFS-141338.

[7] Ganie, Abdul Haseeb, Surender Singh, and Pradeep Kumar Bhatia,"Some new correlation coefficients of picture fuzzy sets with applications," Neural Computing and Applications, pp. 117, 2020. DOI: 10.1007/s00521-020-04715-y.

[8] Wei, Guiwu, "Picture fuzzy cross-entropy for multiple attribute decision making problems," Journal of Business Economics and Management, vol. 17, no. 4, pp. 491-502, 2016. DOI: 10.3846/16111699.2016.1197147.

[9] Wang, Chunyong, Xiaoqiang Zhou, Huonian Tu, and Shengda Tao, "Some geometric aggregation operators based on picture fuzzy sets and their application in multiple attribute decision making,” Ital. J. Pure Appl. Math vol. 37, pp. 477-492, 2017.

[10] Dutta, Palash, and Silpashree Ganju, "Some aspects of picture fuzzy set," Transactions of A. Razmadze Mathematical Institute, Vol. 172, no. 2, pp. 164-175, 2018. DOI: 10.1016/j.trmi.2017.10.006. 
[11] Dogra, Shovan, and Madhumangal Pal. "Picture fuzzy matrix and its application." Soft Computing 24, pp. 9413-9428, 2020. DOI: $10.1007 / \mathrm{s} 00500-020-05021-4$

[12] Si, Amalendu, Sujit Das, and Samarjit Kar. "Picture fuzzy setbased decision-making approach using Dempster-Shafer theory of evidence and grey relation analysis and its application in COVID-19 medicine selection." Soft Computing, pp. 1-15, 2021. DOI:10.1007/s00500-021-05909-9

[13] Wei, Guiwu, "Picture fuzzy aggregation operators and their application to multiple attribute decision making," Journal of Intelligent \& Fuzzy Systems, vol. 33, no. 2, pp. 713-724, 2017. DOI: $10.3233 /$ JIFS-161798.

[14] Garg, Harish, "Some picture fuzzy aggregation operators and their applications to multicriteria decision-making," Arabian Journal for Science and Engineering, vol. 42, no. 12, pp. 52755290, 2017. DOI: 10.1007/s13369-017-2625-9.

[15] Wei, Guiwu, "Picture fuzzy Hamacher aggregation operators and their application to multiple attribute decision making," Fundamenta Informaticae, vol. 157, no. 3, pp. 271-320, 2018. DOI: $10.3233 /$ FI-2018-1628.

[16] Khan, Saifullah, Saleem Abdullah, and Shahzaib Ashraf, "Picture fuzzy aggregation information based on Einstein operations and their application in decision making," Mathematical Sciences, vol. 13, no. 3, pp. 213-229, 2019. DOI: 10.1007/s40096019-0291-7.

[17] Qiyas, Muhammad, Muhammad Ali Khan, Saifullah Khan, and Saleem Abdullah, "Concept of Yager operators with the picture fuzzy set environment and its application to emergency program selection," International Journal of Intelligent Computing and Cybernetics (2020).

[18] Cuong, Bui Cong, Vladik Kreinovitch, and Roan Thi Ngan, "A classification of representable t-norm operators for picture fuzzy sets," In 2016 eighth international conference on knowledge and systems engineering (KSE), pp. 19-24, IEEE, 2016.

[19] Cuong, Bui Cong, and Van Hai Pham, "Some fuzzy logic operators for picture fuzzy sets," In 2015 seventh international conference on knowledge and systems engineering (KSE), pp. 132137. IEEE, 2015. DOI: 10.1109/KSE.2015.20.

[20] Zadeh, Lotfi Asker, "Fuzzy sets as a basis for a theory of possibility," Fuzzy sets and systems, vol. 1, no. 1, pp. 3-28, 1978.

[21] Wenxiu, Zhang, and Le Huiling, "The Extended Fuzzy Operators and the Fuzzy Truth-Valued Possibility Degree," IFAC Proceedings Volumes, vol. 16, no. 13, pp. 103-106, 1983. DOI: 10.1016/S1474-6670(17)62014-7.
[22] Wang, Ying-Ming, Jian-Bo Yang, and Dong-Ling Xu, "A preference aggregation method through the estimation of utility intervals," Computers \& Operations Research, vol. 32, no. 8, pp. 2027-2049, 2005. DOI: 10.1016/j.cor.2004.01.005.

[23] Van Dinh, Nguyen, and Nguyen Xuan Thao, "Some measures of picture fuzzy sets and their application in multi-attribute decision making," Int. J. Math. Sci. Comput.(IJMSC), vol. 4, no. 3, pp. 23-41, 2018. DOI: 10.5815/ijmsc.2018.03.03.

[24] Garg, Harish, and Kamal Kumar, "Improved possibility degree method for ranking intuitionistic fuzzy numbers and their application in multiattribute decision-making," Granular Computing, vol. 4, no. 2, pp. 237-247, 2019. DOI: $10.1007 / \mathrm{s} 41066-018-$ 0092-7.

[25] Wang, Ying-Ming, Jian-Bo Yang, and Dong-Ling Xu, "Interval weight generation approaches based on consistency test and interval comparison matrices," Applied Mathematics and Computation, vol. 167, no. 1, pp. 252-273, 2005. DOI: 10.1016/j.amc.2004.06.080.

[26] Wei, Cui-Ping, and Xijin Tang, "Possibility degree method for ranking intuitionistic fuzzy numbers," In 2010 IEEE/WIC/ACM International Conference on Web Intelligence and Intelligent Agent Technology, IEEE, vol. 3, pp. 142-145, 2010. DOI: 10.1109/WI-IAT.2010.239.

[27] Kumar, Samir, and Animesh Biswas, "Use of possibility measures for ranking of interval valued intuitionistic fuzzy numbers in solving multicriteria decision making problems," In International Conference on Computational Intelligence, Communications, and Business Analytics, Springer, Singapore, pp. 153167, 2017. DOI: 10.1007/978-981-10-6430-2_13.

[28] Ullah, Kifayat, Harish Garg, Tahir Mahmood, Naeem Jan, and Zeeshan Ali. "Correlation coefficients for T-spherical fuzzy sets and their applications in clustering and multi-attribute decision making." Soft Computing 24, no. 3, pp. 1647-1659, 2020. DOI: 10.1007/s00500-019-03993-6.

[29] Chinnadurai, V., and S. Madhanraj. "Cubic picture fuzzy soft matrices.” J. Math. Comput. Sci. 11, no. 6, pp. 7939-7955, 2021. DOI:10.28919/jmcs/6710.

[30] Sindhu, M. S., M. Ahsan, A. Rafiq, and I. A. Khan. "Multiple criteria decision making based on bipolar picture fuzzy sets and extended TOPSIS.” J. Math. Comput. Sci. 23, no. 1, pp. 49-57, 2021. DOI: $10.22436 /$ jmcs.023.01.05. 